\title{
Comparison of glycaemic control in patients with Type 2 diabetes on basal insulin and fixed combination oral antidiabetic treatment: results of a pilot study
}

\author{
G. De Mattia $\cdot$ O. Laurenti $\cdot$ A. Moretti
}

Received: 18 September 2008/ Accepted: 16 October 2008/Published online: 22 November 2008

(c) Springer-Verlag 2008

\begin{abstract}
This randomised, open-label, two-way crossover study compared the coefficient of variance (CV) of fasting and postprandial blood glucose (FBG and PPBG) with insulin glargine (glargine) versus neutral protamine Hagedorn (NPH) insulin treatment in patients with Type 2 diabetes (T2DM). Patients $(N=20)$ on oral antidiabetic drugs (OADs) were treated with NPH (at bedtime) or glargine (at dinnertime) for 12 weeks of each cross-over treatment period; OADs were continued. The FBG CV was calculated from self-monitored BG values and PPBG using venous blood samples, or continuous glucose monitoring system (CGMS). Both insulins provided similar improvements in glycaemic control; however, PPBG was significantly lower after a standard meal test (performed at 13:00 $\mathrm{h}$ the day after insulin injection) with glargine versus NPH $(p=0.02)$. Thirteen versus 15 patients experienced $\geq 1$ episode of hypoglycaemia with glargine versus NPH. The results suggest that glargine plus OADs is more effective in reducing PPBG fluctuations during the day than NPH plus OADs.
\end{abstract}

Keywords T2DM · Glycaemic control · Insulin glargine · Neutral protamine Hagedorn - Fasting blood glucose . Postprandial blood glucose

Results from this study have been presented as a poster at the American Diabetes Association annual congress 2007 (Diabetes 2007; 56[Suppl 1]: A148 [Abstract 555-P]).

G. De Mattia $(\bowtie) \cdot$ O. Laurenti · A. Moretti

Department of Internal Medicine, University of Rome

"Sapienza", Viale del Policlinico, 155, 00161 Rome, Italy

e-mail: giancarlo.demattia@alice.it

\section{Introduction}

Type 2 diabetes mellitus is a progressive disease in which good glycaemic control is essential to prevent or delay the onset of microvascular or macrovascular complications [1]. Continuous glucose monitoring system (CGMS) determinations show that standard measurements of glycaemic control underestimate the occurrence of hyperglycaemia in real-life in Type 2 diabetes mellitus. Given the microvascular and macrovascular damage caused by fasting and postprandial hyperglycaemia, CGMS provides an excellent tool to evaluate alternative therapeutic strategies to reduce hyperglycaemia blood glucose (BG) excursions [2].

A recent study, under real-life conditions found that the 24-h pharmacodynamic profile of insulin glargine was associated with better CGMS BG profiles with smaller BG excursions during the day compared with neutral protamine Hagedorn (NPH) insulin [3], and a similar study found that switching from NPH insulin to insulin glargine also resulted in improved CGMS profiles [4]. Diet and exercise are initially recommended to improve glycaemic control; however, over time this strategy is usually insufficient to maintain glycaemic control, necessitating the introduction of oral antidiabetic drugs (OADs) [5]. As the disease progresses, management with OADs alone becomes increasingly difficult and the addition of insulin therapy is required [6].

Insulin glargine (LANTUS ${ }^{\circledR}$, sanofi-aventis, Paris, France) is a long-acting human insulin analogue with a smooth action profile and no pronounced peak in action [7] that more closely mimics endogenous basal insulin to provide 24-h cover. Insulin glargine is also associated with a reduced risk of hypoglycaemia compared with the traditionally used, intermediate-acting, NPH insulin [8]. Although the effect of insulin glargine on fasting blood 
glucose (FBG) is well characterised in Type 2 diabetes mellitus, less is known about its effects on postprandial glucose handling. Therefore, the aim of the present study was to determine either by CGMS or by venous plasma glucose excursion measurement the relative impact of insulin glargine and NPH insulin on FBG and postprandial glucose handling after a mixed meal in patients with Type 2 diabetes mellitus.

Here, we report results of a pilot study of insulin glargine versus NPH insulin, both in combination with OADs, in a two-way cross-over study to determine their effects on glucose variability after 12 weeks of treatment.

\section{Methods}

\section{Objectives}

The primary objective of this study was to evaluate the coefficient of variability (CV) of FBG calculated from selfmonitored blood glucose (SMBG) values.

The secondary objectives of the study included: glycaemic control (including measurements of glycated haemoglobin $\left[\mathrm{HbA}_{1 \mathrm{c}}\right]$ and $\mathrm{FBG}$ levels); hypoglycaemia; changes in body weight, final insulin dose and lipid profile; and profiles of patients that best fitted each of the algorithms with the dependent variable of change in $\mathrm{HbA}_{1 \mathrm{c}}$ and independent variables, such as age, gender, race, tobacco use, diabetes complications, initial $\mathrm{HbA}_{1 \mathrm{c}}$, initial weight, duration of diabetes mellitus, general education and diabetes education. Safety was assessed by the monitoring of adverse events and other routine laboratory parameters.

\section{Study design}

This was an open-label, national, single-centre, randomised, controlled, two-way cross-over exploratory study. The study comprised a 1-week run-in phase, followed by two 12-week treatment phases and a 2-week safety followup phase (Fig. 1).
Study endpoints

The primary endpoint was FBG CV calculated from SMBG values obtained during the last 4 weeks before Visit (V)3 (end of Treatment Phase 1) and V4 (end of Treatment Phase 2). The secondary endpoints were glycaemic control, as measured by $\mathrm{HbA}_{1 \mathrm{c}}, \mathrm{FBG}$, insulin and $\mathrm{C}$-peptide. In addition, glycaemic control was measured by the glucose levels after a standard meal test determined from venous blood samples or using a CGMS (Glucoday ${ }^{\circledR}$, A.Menarini Diagnostics, Florence, Italy). Other parameters investigated were changes in urinary albumin-to-creatinine ratio; changes in lipid profiles (serum total cholesterol, lowdensity lipoprotein cholesterol, high-density lipoprotein cholesterol and triglycerides); frequency of hypoglycaemia; general safety and any adverse events reported by patients at each visit; and changes in weight and final insulin dose as documented throughout treatment cycles. The analysis of $\mathrm{HbA}_{1 \mathrm{c}}$, plasma insulin, C-peptide, glucagon and free fatty acids was performed at Exacta Lab (Verona, Italy).

\section{Patients}

The primary inclusion criteria included male or female patients $\geq 45$ years old with a diagnosis of Type 2 diabetes mellitus (duration $\geq 5$ years); treatment with OADs in fixed combination (glibenclamide [2.5 mg] + metformin [400 mg]; two or three tablets per day) at a stable dose in the last 3 months; $\mathrm{HbA}_{1 \mathrm{c}} \geq 8$ and $\leq 11 \%$; body mass index $>27$ and $<35 \mathrm{~kg} / \mathrm{m}^{2}$; and willingness and ability to inject insulin and perform SMBG. The primary exclusion criteria included patients diagnosed with Type 1 insulin-dependent diabetes mellitus; patients with fasting C-peptide levels $<1 \mathrm{ng} / \mathrm{ml}$ (to potentially exclude patients with latent autoimmune diabetes of adults); cardiac status New York Heart Association IIIIV; impaired renal function as shown by (but not limited to) serum creatinine $\geq 1.5 \mathrm{mg} / \mathrm{dl}$ for males or $\geq 1.4 \mathrm{mg} / \mathrm{dl}$ for females; and planned pregnancy, pregnant or lactating females. The study was conducted in accordance with the
Fig. 1 Study design and visit schedule. $V$ visit, $p t s$ patients, $O A D$ oral antidiabetic drugs, $H b A_{l c}$ haemoglobin A1c, $N P H$ neutral protamine Hagedorn insulin, $C G M S$ continuous glucose monitoring system

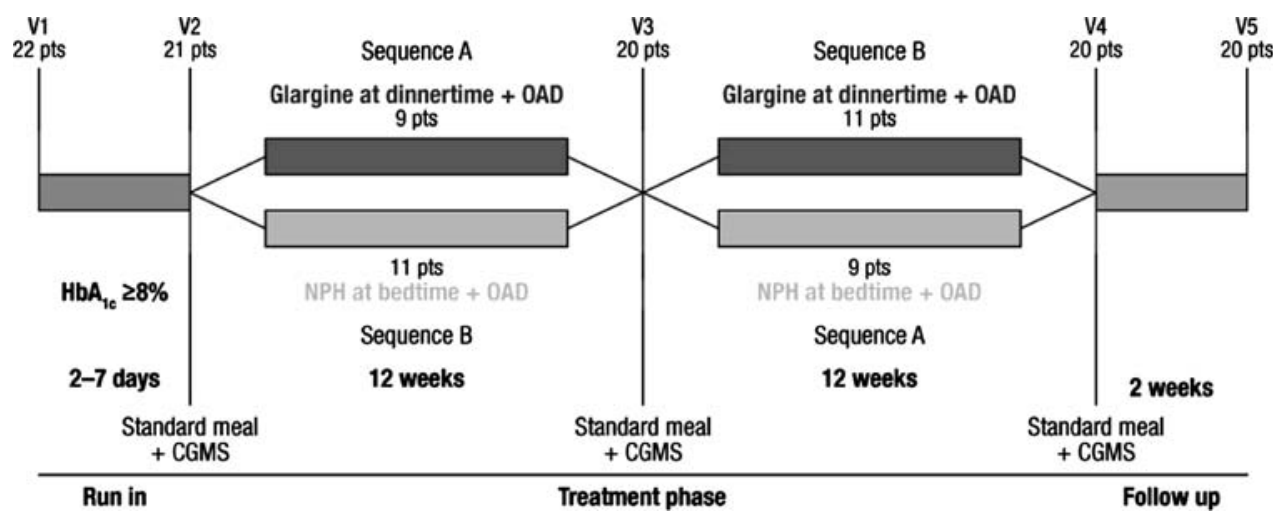


Declaration of Helsinki. Approval by an institutional ethics committee was obtained. All patients provided written informed consent prior to study entry.

\section{Study protocol}

After a standard clinical evaluation (V1), eligibility was confirmed and patients were given standardised diet instructions at study entry. At V2 (baseline), patients were randomised to either Sequence A (insulin glargine followed by NPH insulin) or Sequence B (NPH insulin followed by insulin glargine) (Fig. 1). Study drugs were crossed over after 12 weeks of treatment (V3), followed by a fourth visit (V4) performed at the end of the second 12week treatment cycle. A final follow up (V5) was undertaken 2 weeks after the end of the second 12-week treatment phase (Fig. 1). The starting insulin glargine/NPH insulin dose was $10 \mathrm{IU} /$ day and was titrated every 3 days according to SMBG levels (target FBG: $<100 \mathrm{mg} / \mathrm{dl}$ ), using a modified algorithm (Table 1) based on the Treat-toTarget study [9].

At V2, V3 and V4, participants were fitted with the CGMS device and underwent a mixed meal test, comprising $350 \mathrm{kCal}$ (55\% carbohydrates, 26\% lipids, $19 \%$ proteins) at 13:00 h. Venous blood samples were taken before the meal and 30,60 and $120 \mathrm{~min}$ after the end of the meal, in addition to CGMS monitoring. Throughout the study, SMBG was performed by patients using a glucometer stick test. Measurements of glycaemia in the $48 \mathrm{~h}$ after V2, V3 and V4 were performed by CGMS.

Table 1 Insulin titration algorithm

\begin{tabular}{ll}
\hline Mean FBG $(\mathrm{mg} / \mathrm{dl})$ & Change in insulin dose (U/IU) \\
\hline$>180$ & +6 \\
$160-180$ & +5 \\
$140-159$ & +4 \\
$120-139$ & +2 \\
$100-119$ & +1 \\
$70-99$ & No change \\
$<70$ & -2 \\
\hline
\end{tabular}

The starting insulin glargine/NPH insulin dose was $10 \mathrm{U} / \mathrm{IU}$ per day. The dose of insulin glargine/NPH insulin was to be titrated every 3 days according to the SMBG level, based on the mean FBG value over the last 2 days. Titration was performed using the algorithm presented in Table 1. Up-titration was to be stopped temporarily for 1 week in the event of severe hypoglycaemia, unless there was an explanation for the event (e.g. omission of a meal). Insulin glargine was to be administered at dinnertime; NPH insulin was to be administered at bedtime

$N P H$ neutral protamine Hagedorn, $S M B G$ self-monitored blood glucose, $F B G$ fasting blood glucose
Statistical analysis

The efficacy and safety analyses were performed in the intent-to-treat population. Descriptive summary statistics (number of patients, mean, standard deviation, minimum, maximum) were provided for quantitative variables, while frequency (absolute and relative) distributions were provided for categorical variables. The continuous variables recorded at V2, V3 and V4 were analysed using an analysis of variance (ANOVA) according to a cross-over design, with period and treatment effect. Multiple comparisons were calculated for the general mean of the baseline insulin glargine and NPH insulin values. The comparisons were performed compared with the baseline mean and between treatments and are reported with the corresponding 95\% confidence interval (CI). For each parameter recorded during the meal test, the area under the curve (AUC) adjusted for the basal value (time 0) was calculated according to the trapezoidal rule and analysed with the same cross-over model used in the ANOVA applied to the main parameters. Multiple comparisons were also carried out with the related $95 \%$ CI.

\section{Results}

Study population

Twenty-one patients were randomised to receive the assigned treatment, of whom ten were assigned to Sequence A (insulin glargine followed by NPH insulin) and 11 were assigned to Sequence B (NPH insulin followed by insulin glargine). One patient assigned to Sequence A discontinued the study at V2 owing to consent withdrawal. Therefore, 20 patients completed the total study period (9 in Sequence A and 11 in Sequence B). The baseline characteristics were similar between the treatment groups (Table 2).

Table 2 Baseline characteristics of 20 patients completed

\begin{tabular}{ll}
\hline Characteristic & \\
Males/females $(n[\%])$ & $14(70) / 6(30)$ \\
Age (years) & $59.4 \pm 8.2$ \\
Weight $(\mathrm{kg})$ & $82.7 \pm 8.7$ \\
Body mass index $\left(\mathrm{kg} / \mathrm{m}^{2}\right)$ & $29.5 \pm 2.0$ \\
$\mathrm{HbA}_{1 \mathrm{c}}(\%)$ & $9.3 \pm 1.4$ \\
Fasting blood glucose $(\mathrm{mg} / \mathrm{dl})$ & $203.6 \pm 58.3$ \\
\hline
\end{tabular}

All data are mean \pm standard deviation unless otherwise stated $H b A_{1 c}$ haemoglobin $\mathrm{A}_{1 \mathrm{c}}$ 
Fig. 2 a Fasting blood glucose as measured by self-monitoring at baseline and at endpoint. Results are means \pm coefficient of variation. $C V$ coefficient of variation, $F B G$ fasting blood glucose, $N P H$ neutral protamine Hagedorn. b $\mathrm{HbA}_{1 \mathrm{c}}$ levels across both study drugs. $H b A_{l c}$ haemoglobin A1c, $N P H$ neutral protamine Hagedorn;

$* * p<0.01$ versus baseline

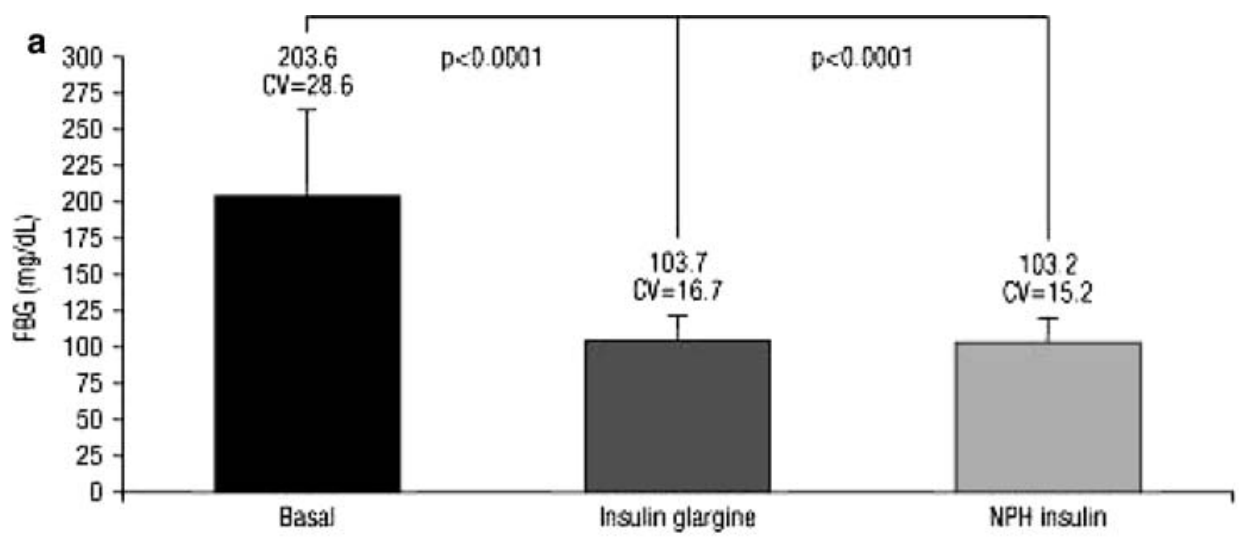

b

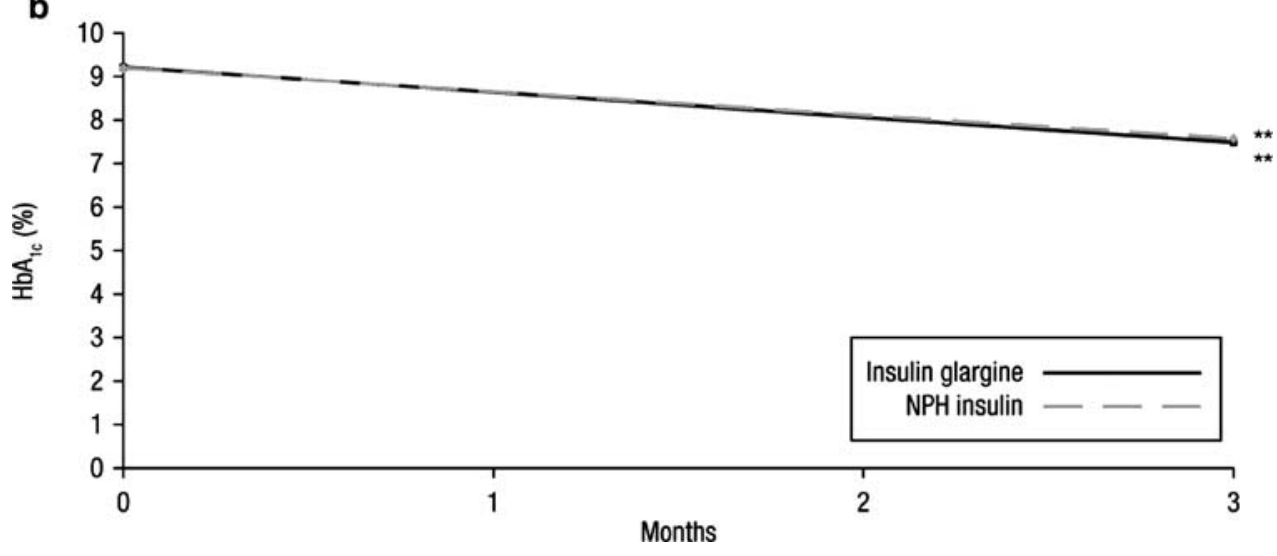

Glycaemic control

Over the duration of the study, the decrease in FBG values was significant for both study therapies $(p<0.0001$ for both) with no significant differences between the two insulins $(p=0.95)$ (Fig. 2a). $\mathrm{HbA}_{1 \mathrm{c}}$ decreased significantly with both insulin glargine (mean \pm standard deviation [SD]: $-1.7 \pm 1.6 \%)$ and NPH insulin $(-1.6 \pm 1.6 \%)$ compared with baseline ( $p<0.0001$ for both) (Fig. 2b). The mean amplitude of glucose excursions (MAGE) index measured in the SMBG tended to improve between baseline and endpoint with both insulin glargine $(-17.0 \mathrm{mg} / \mathrm{dl} ; 95 \% \mathrm{CI}:-34.5$, $0.6 \mathrm{mg} / \mathrm{dl} ; p=0.058)$ and with NPH insulin $(-13.1 \mathrm{mg} / \mathrm{dl}$; 95\% CI: $-31.4,5.3 \mathrm{mg} / \mathrm{dl} ; p=0.152)$, but there was no difference between the two insulins ( $p=0.603$ ). Mean daily BG (MDBG) measured in the SMBG improved significantly between baseline and endpoint with both insulin glargine $(-40.9 \mathrm{mg} / \mathrm{dl} ; 95 \% \mathrm{CI}:-57.0,-24.8 \mathrm{mg} / \mathrm{dl} ; p<0.0001)$ and with NPH insulin $(-43.9 \mathrm{mg} / \mathrm{dl} ; 95 \% \mathrm{CI}:-59.9$, $-27.8 \mathrm{mg} / \mathrm{dl} ; \quad p<0.0001)$ : there were no differences between the two insulins $(p=0.701)$.

\section{Meal test}

The CGMS-determined post-meal test BG profile revealed a lower BG excursion with insulin glargine than with NPH insulin $(p<0.05$ at 5 -h post-meal test and $p<0.01$ at 6 -h post-meal test; Fig. 3a). In response to the standard meal test, postprandial BG control was better overall with insulin glargine, with a significantly lower AUC at endpoint $(p=0.02)$ compared with NPH insulin (Fig. 3b). The plasma insulin AUC decreased between baseline and endpoint with both insulin glargine $(-61.5 \mathrm{mU} / 1 \mathrm{~min} ; 95 \% \mathrm{CI}$ : $-97.2,-25.7 \mathrm{mU} / \mathrm{lmin} ; p=0.002)$ and $\mathrm{NPH}$ insulin $(-31.8 \mathrm{mU} / 1 \mathrm{~min} ; 95 \% \mathrm{CI}:-66.5,2.9 \mathrm{mU} / 1 \mathrm{~min} ; p=$ $0.070)$, the magnitude of which tended to be greater with insulin glargine, although this was not significant $(p=$ 0.109). The plasma glucagon AUC ( $\mu \mathrm{g} / \mathrm{l} \mathrm{min}$ ) was similar with insulin glargine and NPH insulin (mean \pm SD: $148.7 \pm 12.6$ vs. $153.5 \pm 12.4 \mu \mathrm{g} / \mathrm{l} \mathrm{min} ; p=0.3682)$, and was unchanged from baseline $(146.4 \pm 12.3 \mu \mathrm{g} / \mathrm{l} \mathrm{min}$; $p>0.05$ for both). The plasma C-peptide AUC increased between baseline and endpoint with both insulin glargine $(+17.6 \mu \mathrm{g} / \mathrm{l} \mathrm{min} ; 95 \% \mathrm{CI}:-3.4,38.7 \mu \mathrm{g} / \mathrm{l} \mathrm{min} ; p=0.096)$ and with $\mathrm{NPH}$ insulin $(+39.1 \mu \mathrm{g} / \mathrm{l} \mathrm{min} ; 95 \% \mathrm{CI}: 16.0$, $62.2 \mu \mathrm{g} / \mathrm{l} \mathrm{min} ; p=0.002)$. Although the change tended to be greater with NPH insulin, this was not significant ( $p=0.090$ ).

Insulin therapy

The total daily dose at endpoint was $28.8 \mathrm{U}$ versus $34.7 \mathrm{IU}$ for insulin glargine versus NPH insulin, respectively. 
Fig. 3 a Continuous glucose monitoring system profiles in response to the meal test. $B L$ before lunch, $N S$ nonsignificant, $A L$ after lunch, $N P H$ neutral protamine Hagedorn. b Meal test blood glucose levels after each treatment. Results are means \pm coefficient of variation. $A U C$ area under the curve, $C V$ coefficient of variation, $N P H$ neutral protamine Hagedorn
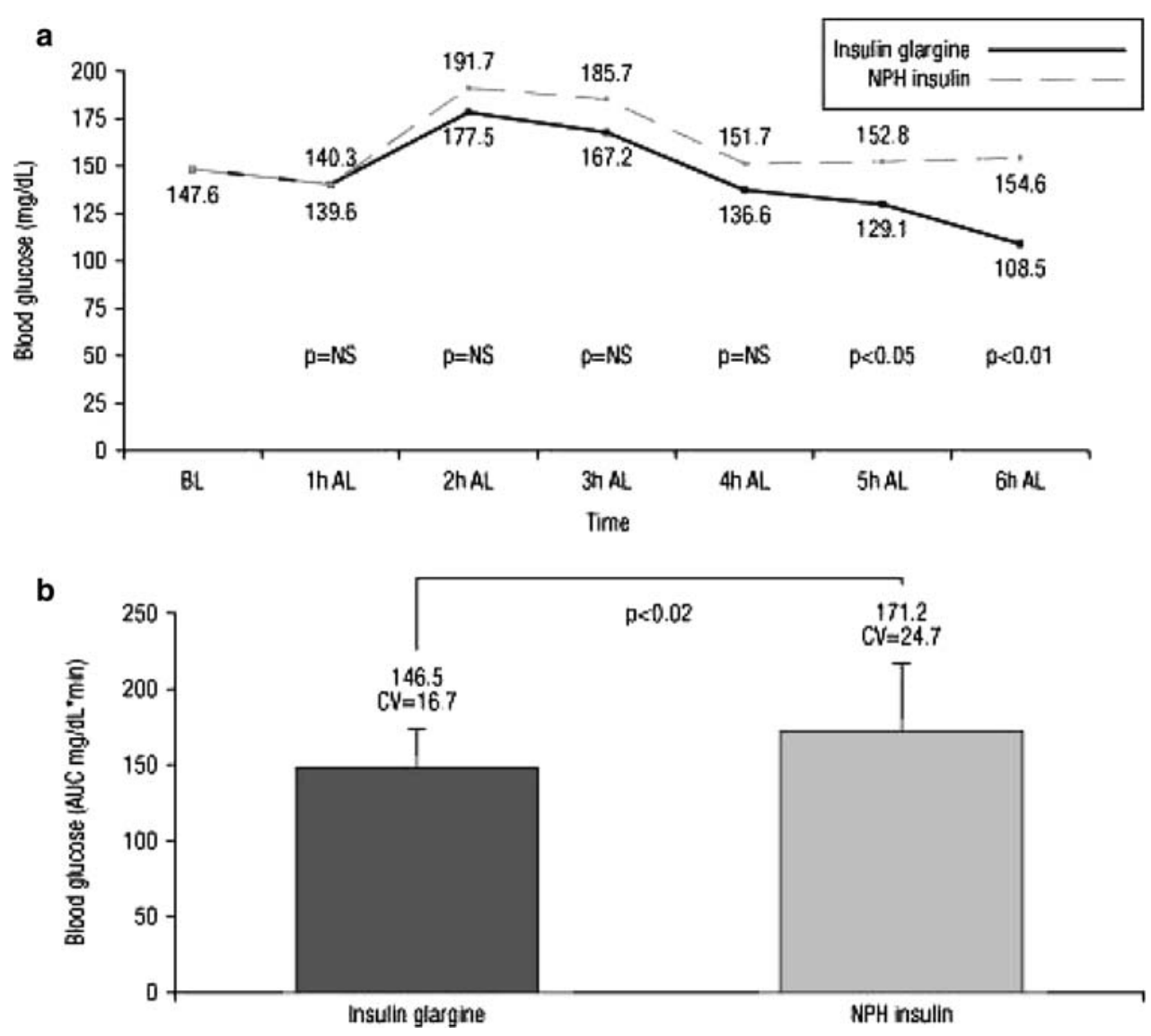

Hypoglycaemia and safety

Thirteen insulin glargine-treated patients and $15 \mathrm{NPH}$ insulin-treated patients experienced at least one episode of hypoglycaemia during treatment. Of those patients receiving insulin glargine, four patients had one episode, three patients had two episodes, two patients had three episodes and four patients had more than five episodes of hypoglycaemia. Among patients receiving NPH insulin, one patient had one episode, two patients had two episodes, three patients had three episodes, two patients had five episodes and seven patients had more than five episodes. None of the episodes in either treatment group was considered to be severe. Overall, the incidence of hypoglycaemia was lower with insulin glargine versus NPH insulin (1.04 vs. 2.12 episodes/patient per month).

Three patients experienced at least one adverse event during treatment with insulin glargine. None of the events was considered to be related to study drug.

Other secondary parameters

No significant changes were observed in either treatment group at any time point for other secondary parameters, including body weight, haematology or blood chemistry.

\section{Discussion}

This study demonstrates that the initiation of insulin therapy with OADs effectively improves glycaemic control in patients with Type 2 diabetes mellitus. Both insulins achieved similar improvements in FBG, MAGE, MDBG and $\mathrm{HbA}_{1 \mathrm{c}}$ over 12 weeks of treatment, with comparable improvements in within-patient variability, and supporting the perception that there was no clinically different impact on glycaemic control. Aside from the overall measures of glycaemic control, participants in our study also underwent standard meal tests (at 13:00 h), at baseline and after each 12-week treatment period.

Although both treatments provided comparable improvements in glycaemic control, blood glucose AUCs were significantly higher with NPH insulin (administered once daily at bedtime) compared with insulin glargine (administered once daily at dinnertime). Taking into account the cross-over study design, these results indicate that insulin glargine may provide better control of the postprandial glucose levels, as measured by the meal test. This finding may be related to a waning of the effect of NPH insulin, which was administered at bedtime (approximately 22:00 h), owing to its duration of action of 12-18 h [7] and rate of subcutaneous absorption [10]. It may be that, if 
NPH insulin had been administered twice daily, the postmeal glucose levels may have more closely matched those achieved with insulin glargine.

Owing to the relatively small sample size of our study (20 patients completed both arms of the study), and the fact that there is some evidence for a carry-over effect after cross-over, results presented here should be interpreted with caution. However, the results of this pilot study warrant prospective evaluation in a larger population of patients, which could be powered based on the magnitude of differences between the two treatment groups. Further studies are warranted using a larger patient population to better compare the differences in post-meal glucose handling between insulin glargine and NPH insulin to better define the qualitative aspect of each $\mathrm{HbA}_{1 \mathrm{c}}$ level detected at the end of each treatment. It is clear from our results that insulin glargine provides significant improvements in glycaemic control at lunchtime compared with NPH insulin. Nevertheless, management of the fasting and postprandial components of glycaemic control is important owing to their contributions to overall glycaemic control [11]. The introduction of short-acting insulin at mealtimes may help these patients achieve their glycaemic goals. It may be that, with better understanding of the impact of insulin glargine on postprandial glycaemia, the administration of prandial insulin at the meal associated with the highest glucose excursion may provide the required improvements in glycaemic control [12]. Indeed, this was partly evaluated in the Orals Plus insulin glulisine and insulin glargine (OPAL) study, in which patients treated with insulin glargine plus OADs were randomised to receive once-daily insulin glulisine at either breakfast or the main meal (defined as the meal associated with the largest prandial glucose excursion). In that study, the improvements in glycaemic control were comparable in both treatment groups (equivalence of breakfast versus main meal administration was shown), but there was a tendency for more patients in the main meal versus breakfast group to reach $\mathrm{HbA}_{1 \mathrm{c}} \leq 6.5 \%$ (33.8 vs. 27.8\%) [13]. Accordingly, future studies could evaluate the postprandial glucose excursions using CGMS in a 'real-life' situation of patients treated with insulin glargine at dinnertime or bedtime. The lower blood glucose fluctuations during the day with glargine versus NPH can be detected by additional qualitative measurements of glycaemic control or new CGMS measurements on top of traditional quantitative $\mathrm{HbA}_{1 \mathrm{c}}$ values [14]. In fact, a recent study conducted in patients with diabetes by Nathan and colleagues found that a linear relationship exists between $\mathrm{HbA}_{1 \mathrm{c}}$ levels and average glucose (AG), as measured by CGMS in a clinically relevant range of glycaemia [15].

The control of glycaemic levels by limiting the magnitude of hyperglycaemia spikes (Fig. 3a) or hypoglycaemia troughs in the management of insulin therapy of Type 2 diabetes mellitus patients can prevent the development of long-term diabetes complications, such as cardiovascular disease, nephropathy, retinopathy and neuropathy [16-18]. The quantitative measurement of $\mathrm{HbA}_{1 \mathrm{c}}$ levels is a widely used, reliable, simple and easy method for assessing glycaemic control $[17,19,20]$. Indeed, current diabetes guidelines have delineated target $\mathrm{HbA}_{1 \mathrm{c}}$ levels to prevent long-term diabetic complications [16-18, 21]. However, measuring glycaemic control by utilising standard measurements of $\mathrm{HbA}_{1 \mathrm{c}}$ levels can limit, by masking the true nature of glycaemic variability, the clear interpretation of biological efficiency of insulin treatment from a long-term perspective $[22,23]$ for people with diabetes and especially for those with Type 2 diabetes mellitus [24].

In conclusion, the results indicate that adding insulin glargine to existing OADs is more effective in reducing postprandial BG fluctuations during the day compared with NPH insulin plus OADs, with a lower incidence of hypoglycaemia.

Acknowledgments This study was supported by sanofi-aventis. Editorial support for this manuscript was provided by the Global Publications group of sanofi-aventis.

Conflict of interest statement The authors have received funding from sanofi-aventis for consulting and speaking, as well as reimbursement for attending a symposium.

\section{References}

1. Davidson JA (2004) Treatment of the patient with diabetes: importance of maintaining target hba(1c) levels. Curr Med Res Opin 20:1919-1927

2. Praet SF, Manders RJ, Meex RC et al (2006) Glycaemic instability is an underestimated problem in type II diabetes. Clin Sci (Lond) 111:119-126

3. Wang XL, Lu JM, Pan CY et al (2007) Evaluation of the superiority of insulin glargine as basal insulin replacement by continuous glucose monitoring system. Diabetes Res Clin Pract 76:30-36

4. Zick R, Petersen B, Richter M, Haug C (2007) Comparison of continuous blood glucose measurement with conventional documentation of hypoglycemia in patients with type 2 diabetes on multiple daily insulin injection therapy. Diabetes Technol Ther 9:483-492

5. Chehade JM, Mooradian AD (2000) A rational approach to drug therapy of type 2 diabetes mellitus. Drugs 60:95-113

6. Rosenstock J (2004) Basal insulin supplementation in type 2 diabetes; refining the tactics. Am J Med 116(Suppl 3A):10S-16S

7. Lepore M, Pampanelli S, Fanelli C et al (2000) Pharmacokinetics and pharmacodynamics of subcutaneous injection of long-acting human insulin analog glargine, NPH insulin, and ultralente human insulin and continuous subcutaneous infusion of insulin lispro. Diabetes 49:2142-2148

8. Mullins P, Sharplin P, Yki-Jarvinen H, Riddle MC, Haring HU (2007) Negative binomial meta-regression analysis of combined glycosylated hemoglobin and hypoglycemia outcomes across 
eleven phase III and IV studies of insulin glargine compared with neutral protamine Hagedorn insulin in type 1 and type 2 diabetes mellitus. Clin Ther 29:1607-1619

9. Riddle MC, Rosenstock J, Gerich J (2003) The treat-to-target trial: randomized addition of glargine or human $\mathrm{nph}$ insulin to oral therapy of type 2 diabetic patients. Diabetes Care 26:30803086

10. Luzio SD, Beck P, Owens DR (2003) Comparison of the subcutaneous absorption of insulin glargine (LANTUS) and NPH insulin in patients with type 2 diabetes. Horm Metab Res 35:434438

11. Monnier L, Colette C (2006) Contributions of fasting and postprandial glucose to hemoglobin a1c. Endocr Pract 12(Suppl 1):42-46

12. Monnier L, Colette C (2006) Addition of rapid-acting insulin to basal insulin therapy in type 2 diabetes: indications and modalities. Diabetes Metab 32:7-13

13. Lankisch LM, Ferlinz KC, Leahy JL et al (2008) Introducing a simplified approach to therapy in type 2 diabetes: a comparision of two single-dose regimens of insulin glulisine plus insulin glargine and oral antidiabetic drugs. Diabetes Obes Metab. doi: 10.1111/j.1463-1326.2008.00967.x

14. Service FJ, O'Brien PC (2001) The relation of glycaemia to the risk of development and progression of retinopathy in the diabetic control and complications trial. Diabetologia 44:1215-1220

15. Nathan DM, Kuenen J, Borg R et al (2008) Translating the a1c assay into estimated average glucose values. Diabetes Care $31: 1473-1478$
16. A desktop guide to type 2 diabetes mellitus (1999) European diabetes policy group 1999. Diabetes Med 16:716-730

17. ADA (2007) Standards of medical care in diabetes-2007. Diabetes Care 30(Suppl 1):S4-S41

18. NICE (2008) The Royal College of General Practitioners Effective Clinical Practice Unit: clinical guidelines for type 2 diabetes management of blood glucose. Available at: http://www.Nice. Org.Uk/nicemedia.Pdf. Accessed October 2008

19. Goldstein DE, Little RR, Lorenz RA et al (2004) Tests of glycemia in diabetes. Diabetes Care 27:1761-1773

20. Saudek CD, Derr RL, Kalyani RR (2006) Assessing glycemia in diabetes using self-monitoring blood glucose and hemoglobin a1c. JAMA 295:1688-1697

21. Nathan DM, Buse JB, Davidson MB et al (2006) Management of hyperglycaemia in type 2 diabetes: a consensus algorithm for the initiation and adjustment of therapy: a consensus statement from the American Diabetes Association and the European Association for the Study of Diabetes. Diabetologia 49:1711-1721

22. Gaede P, Vedel P, Larsen N et al (2003) Multifactorial intervention and cardiovascular disease in patients with type 2 diabetes. N Engl J Med 348:383-393

23. DCCT/EDIC (2002) Effect of intensive therapy on the microvascular complications of type 1 diabetes mellitus. JAMA 287:2563-2569

24. Hirsch IB (2008) Piecing the puzzle together: according to whom? J Clin Endocrinol Metab 95:1161-1163 